\title{
Determination of the stationary state densities of the stochastic nonlinear dynamical systems
}

\author{
Serkan Günel ${ }^{\text {a }}$, F. Acar Savaci ${ }^{\text {b,* }}$ \\ ${ }^{a}$ Katholieke Universiteit Leuven, ESAT-SISTA, Kasteelpark Arenberg 10, 3001 Leuven(Heverlee), Belgium \\ b İzmir Institute of Technology, Department Electrical and Electronics Engineering, Urla/İzmir, Turkey
}

Received 5 May 2005; received in revised form 5 February 2006; accepted 10 June 2006

Available online 10 October 2006

(Communicated by H. DEMIRAY)

\begin{abstract}
The stationary state probability densities appear not only in the study of dynamical systems with random vector fields, but also in the deterministic dynamical systems exhibiting chaotic behavior when the uncertainties in the initial conditions are represented with the probability densities. But since it is very hard problem to determine these densities, in this paper the new efficient method to obtain an approximate solution of Fokker-Planck-Kolmogorov equation which arises in the determination of the stationary state probability densities has been given by representing the densities with compactly supported functions. With specific choice of the compactly supported functions as piecewise multivariable polynomials which are supported on the ellipsoidal regions, the parameters to be calculated for determining the densities can be considerably decreased compared to Multi-Gaussian Closure scheme, in which the stationary densities are assumed to be the weighted average of the Gaussian densities. The main motivation to choose the compactly supported functions is that, in the chaotic dynamics the states are trapped in a specific compact subspace of the state space. The stationary state densities of two basic examples commonly considered in the literature have been estimated using the Parzen's estimator, and the densities obtained using the newly proposed method have been compared with these estimated densities and the densities obtained with the Multi-Gaussian Closure scheme. The results indicate that the presented compactly supported piecewise polynomial scheme can be successful compared to Multi-Gaussian scheme, when the system is highly nonlinear.
\end{abstract}

(c) 2006 Elsevier Ltd. All rights reserved.

Keywords: Random dynamical systems; Fokker-Planck-Kolmogorov equation; Compactly supported multivariable polynomials

\section{Introduction}

The effect of indeterministic inputs on the dynamical systems can be considered in the view of FokkerPlanck-Kolmogorov (FPK) formalism. This formalism gives one the ability to transform the random

\footnotetext{
* Corresponding author. Tel.: +90 23275065 11; fax: +90 2327506505.

E-mail addresses: serkan.gunel@esat.kuleuven.be (S. Günel), acarsavaci@iyte.edu.tr (F.A. Savac1).
} 
dynamical systems evolving in the state space under the influence of Gaussian white noise either in additive or in multiplicative form, to a new dynamical system evolving in the space of probability density functions stated in terms of a linear parabolic partial differential equation, the Fokker-Planck-Kolmogorov equation [1]. Such probability densities appear not only in the study of dynamical systems with random vector fields, but also in the deterministic dynamical systems exhibiting chaotic behavior when the uncertainties in the initial conditions are represented with the densities [2].

It is known that an exact solution of the FPK equation is generally hard to find and only in some special cases exact solution is known [3-8]. Many approximate methods have been developed, such as the variational methods based on the eigenfunction expansion of the probability density function (pdf) [9,10], iterative methods based on the solution of an integral equation [1,11], the maximum entropy approach originated by the classical work of Jaynes [12] in which the solution of the FPK equation is presented by an infinite dimensional dynamical system of the moments of the pdfs [13,14] and weighted residual schemes [15-19]. A simple approach is the Gaussian Closure (GC) method where the solution is assumed to be Gaussian and the parameters of the Gaussian pdf are chosen in order to minimize the approximation error [20]. This approach has been extended to Multi-Gaussian case in which the solution is assumed to be the sum of Gaussian pdfs [21]. Another variant of this method called Exponential Closure is to use the exponential functions of polynomials of the state variables, instead of Gaussian assumption $[16,17]$. In these methods, solution of the FPK equation in the weak sense is reduced to a solution of nonlinear algebraic equations.

The GC is unsuitable when the system is highly nonlinear or when multiplicative random excitations exist. In order to eliminate these drawbacks, the MGC method is used, but in this case the number of free parameters to be determined can be quite large, consequently, the method may not be feasible for high dimensional nonlinear systems.

In Section 2.1 of this paper, the FPK equation arising from the stochastic dynamical system driven with Gaussian white noise has been introduced first. In Section 2.2, the existing methods to obtain the approximate solution of the FPK equation have been summarized. In Section 2.3 the new method based on the weighted residual scheme has been proposed, in which instead of the exponential functions of polynomials, compactly supported multivariable polynomial functions (CSP) have been used to simplify the problem of obtaining and solving nonlinear algebraic equations which represent the FPK equation. Then in Section 3, to clarify the usefulness of this newly proposed method, the stationary state densities of dynamical systems with cubic nonlinearities whose exact solutions are known, have been obtained and the results have been compared with the densities obtained by the MGC scheme and the estimated densities obtained using the Parzen's kernel density estimator $[22,23]$.

\section{The Fokker-Planck-Kolmogorov formalism}

\subsection{The Fokker-Planck-Kolmogorov equation}

Let $L^{m}\left(\mathbb{R}^{n}\right)$ denote the measure space consists of all integrable functions $f: \mathbb{R}^{n} \rightarrow \mathbb{R}$, such that

$$
\int_{\mathbb{R}^{n}}|f(\mathbf{x})|^{m} \mathrm{~d} \mathbf{x}<\infty
$$

and $D\left(\mathbb{R}^{n}\right) \subset L^{1}\left(\mathbb{R}^{n}\right)$ be a set of density functions defined by

$$
D\left(\mathbb{R}^{n}\right) \triangleq\left\{p \in L^{1}\left(\mathbb{R}^{n}\right) \mid p \geqslant 0 \text { and }\|p\|_{L^{1}}=1\right\} .
$$

Consider the following stochastic differential equation:

$$
\frac{\mathrm{d} \mathbf{x}}{\mathrm{d} t}=f(\mathbf{x})+g(\mathbf{x}) \eta
$$

with initial condition

$$
\mathbf{x}(0)=\mathbf{x}_{0},
$$


where $t \geqslant 0, \quad \mathbf{x}=\left(x_{1} x_{2} \cdots x_{n}\right)^{\mathrm{T}} \in \mathbb{R}^{n}, f(\mathbf{x}) \in \mathbb{R}^{n}, \quad g(\mathbf{x}) \in \mathbb{R}^{n \times n}$ and $\eta(t)$ is the white noise defined as $\eta(t) \triangleq\left(\frac{\mathrm{d} \omega_{1}}{\mathrm{~d} t} \frac{\mathrm{d} \omega_{2}}{\mathrm{~d} t} \cdots \frac{\mathrm{d} \omega_{n}}{\mathrm{~d} t}\right)$, where $\omega_{i}$ are 1-D Wiener processes, $E\left\{\omega_{i}(t)\right\}=0, \quad E\left\{\omega_{i}(t) \omega_{i}(t+\tau)\right\}=2 \delta(\tau)$, $i=1, \ldots, n$ and $E\{\cdot\}$ denotes the expectation operator.

The density function $p(\mathbf{x}, t) \in D\left(\mathbb{R}^{n}\right)$ of the states $\mathbf{x}$, at time $t$ can be defined as

$$
\operatorname{prob}\left\{\mathbf{x}(t) \in B \subset \mathbb{R}^{n}\right\}=\int_{B} p(\mathbf{z}, t) \mathrm{d} \mathbf{z}
$$

where $\operatorname{prob}\{\cdot\}$ denotes the probability and $p(\mathbf{x}, t)$ satisfies

$$
\lim _{\|\mathbf{x}\| \rightarrow \pm \infty} p(\mathbf{x}, t)=0 \quad \forall t \geqslant 0 .
$$

Theorem (Fokker-Planck-Kolmogorov [2]). If the functions $g_{i j}, \frac{\partial g_{i j}}{\partial x_{k}}, \frac{\partial^{2} g_{i j}}{\partial x_{k} \partial x_{i}}, f_{i}, \frac{\partial f_{i}}{\partial x_{i}}, \frac{\partial p}{\partial t}, \frac{\partial p}{\partial x_{i}}, \frac{\partial^{2} p}{\partial x_{i} \partial x_{j}}$ are continuous for $t>0$ and $\mathbf{x} \in \mathbb{R}^{n}$, and if $f_{i}, g_{i j}$ and their first derivatives are bounded, then $p(\mathbf{x}, t) \in D\left(\mathbb{R}^{n}\right)$ satisfies

$$
\frac{\partial p}{\partial t}=\frac{1}{2} \sum_{i, j=1}^{n} \frac{\partial^{2}}{\partial x_{i} \partial x_{j}}\left(a_{i j} p\right)-\sum_{i=1}^{n} \frac{\partial}{\partial x_{i}}\left(f_{i} p\right) \quad \forall t>0, \mathbf{x} \in \mathbb{R}^{n}
$$

with initial condition

$$
p(\mathbf{x}, 0)=p_{0}(\mathbf{x}), \quad p_{0}(\mathbf{x}) \in D\left(\mathbb{R}^{n}\right),
$$

where $a_{i j}(\mathbf{x}) \triangleq \sum_{k=1}^{n} g_{i k}(\mathbf{x}) g_{j k}(\mathbf{x}) i, j=1,2, \ldots, n$, which is always nonnegative, and $p_{0}(\mathbf{x})$ is the initial density of the states.

Defining the FPK operator as

$$
\mathscr{L}_{t} \triangleq \frac{1}{2} \sum_{i=1}^{n} \sum_{j=1}^{n} \frac{\partial^{2}}{\partial x_{i} \partial x_{j}}\left(a_{i j}(\mathbf{x})(\cdot)\right)-\sum_{i=1}^{n} \frac{\partial}{\partial x_{i}}\left(f_{i}(\mathbf{x})(\cdot)\right)-\frac{\partial}{\partial t}(\cdot)
$$

and the system given in (1) with the initial density of the states (5), then FPK equation (4) can be written as

$$
\mathscr{L}_{t} p(\mathbf{x}, t)=0 \quad \mathbf{x} \in \mathbb{R}^{n}, t>0 .
$$

The stationary density $p_{\mathrm{st}}(\mathbf{x})$, if exists, is defined by the limit

$$
\lim _{t \rightarrow \infty} p(\mathbf{x}, t) \triangleq p_{\mathrm{st}}(\mathbf{x}) \quad \forall p_{0}(\mathbf{x}) \in D\left(\mathbb{R}^{n}\right) .
$$

In the stationary case, the FPK operator can be defined as

$$
\mathscr{L} \triangleq \frac{1}{2} \sum_{i=1}^{n} \sum_{j=1}^{n} \frac{\partial^{2}}{\partial x_{i} \partial x_{j}}\left(a_{i j}(\mathbf{x})(\cdot)\right)-\sum_{i=1}^{n} \frac{\partial}{\partial x_{i}}\left(f_{i}(\mathbf{x})(\cdot)\right)
$$

and hence, the stationary density should satisfy the reduced FPK equation

$$
\mathscr{L} p_{\text {st }}(\mathbf{x})=0 \quad \forall p_{0}(\mathbf{x}) \in D\left(\mathbb{R}^{n}\right)
$$

together with the boundary condition (3). Multiplying both sides of (10) with an arbitrary test function $h: \mathbb{R}^{n} \rightarrow \mathbb{R}$ of Hilbert space $\mathscr{H}$ with the usual inner product defined by $\langle f(\mathbf{x}), g(\mathbf{x})\rangle \triangleq$ $\int_{\mathbb{R}^{n}} f(\mathbf{x}) g(\mathbf{x}) \mathrm{d} \mathbf{x} \forall f, g \in \mathscr{H}$, and integrating both sides over the whole domain yields the variational equation

$$
\left\langle\mathscr{L} p_{\mathrm{st}}(\mathbf{x}), h(\mathbf{x})\right\rangle=0 .
$$

Clearly, all solutions of (10) are the solutions of the variational equation (11), but the inverse statement, in general, is not true. Finding solution of the infinite dimensional variational problem is almost as demanding as the solution of the original problem. In practice, a finite set of linearly independent test functions, $\mathscr{Y} \triangleq\left\{h_{k} \in \mathscr{H}\right\}_{k=1}^{N}$ are chosen in order to satisfy

$$
\left\langle\mathscr{L} p_{\mathrm{st}}(\mathbf{x}), h_{k}(\mathbf{x})\right\rangle=\int_{\mathbb{R}^{n}} \mathscr{L} p_{\mathrm{st}}(\mathbf{x}) h_{k}(\mathbf{x}) \mathrm{d} \mathbf{x}=0, \quad k=1,2, \ldots, N .
$$


The solution $p_{\mathrm{st}}(\cdot)$, satisfying (12) is called the solution of the reduced FPK equation (10) in the weak sense. The error between the exact solution of the variational problem and the weak sense solution vanishes in the space spanned by $\left\{h_{k}\right\}_{k=1}^{N}$.

\subsection{Approximate solution of the FPK equation}

Let $p_{\vartheta}(\mathbf{x})$ be an estimate of the solution of (10) parameterized by the $N$-dimensional vector $\vartheta$. An approximate stationary solution in the weak sense can be obtained by substituting $p_{\vartheta}(\mathbf{x})$ in the FPK equation, and determining the parameters using the orthogonality requirement of the test functions, and the right hand side of the (10). This yields a set of nonlinear algebraic equations to be solved for $\vartheta$ in terms of system parameters, i.e.

$$
\Upsilon_{k}(\vartheta) \triangleq\left\langle\mathscr{L} p_{\vartheta}(\mathbf{x}), h_{k}\right\rangle=0, \quad k=1,2, \ldots, N .
$$

Denoting the resolution as the number of the basis functions, $K$, and if $p_{\vartheta}(\mathbf{x})$ is chosen to be

$$
p_{\vartheta}(\mathbf{x})=\sum_{i=1}^{K} \frac{\kappa_{i}}{\sqrt{(2 \pi)^{n}\left|\Gamma_{i}\right|}} \mathrm{e}^{-\frac{1}{2}\left(\mathbf{x}-\mathbf{m}_{\mathbf{i}}\right)^{\mathrm{T}} \Gamma_{i}^{-1}\left(\mathbf{x}-\mathbf{m}_{\mathbf{i}}\right)},
$$

where $|\cdot|$ denotes the determinant, $\vartheta=\left\{\kappa_{i}, \Gamma_{i}, \mathbf{m}_{i}, i=1, \ldots, K\right\}, \Gamma_{i}$, and $\mathbf{m}_{i}$ denote $n \times n$ positive symmetric covariance matrices, $n \times 1$ mean vectors to be determined respectively, then the method of obtaining approximate solution is called Multi-Gaussian Closure (MGC) method [21]. The special case where $K=1$, is equivalent to Gaussian-Closure (GC) method. Another choice for $p_{\vartheta}(\mathbf{x})$ is an exponential function of a polynomial $Q_{N}(\mathbf{x})$, such that

$$
\begin{aligned}
& p_{\vartheta}(\mathbf{x})=c \mathrm{e}^{Q_{N}(\mathbf{x})}, \\
& Q_{N}(\mathbf{x})=\sum_{0<\sum_{i=1}^{n} k_{i} \leqslant N} \vartheta_{k_{1}, k_{2}, \ldots, k_{n}} \prod_{j=1}^{n} x_{j}^{k_{j}},
\end{aligned}
$$

where $c$ is a normalization constant selected such that $p_{\vartheta}(\cdot)$ is normalized. In this case, the method is called as Exponential Closure method [24,16,17].

In both cases, the test functions are chosen to be simple monomials or monomials multiplied by the pdf obtained by the GC, in which the approximate pdfs do not have compact support.

However, in practice, the stationary states of the stable systems are trapped in a specific compact subspace of $\mathbb{R}^{n}$, which implies that the integration over the whole domain is unnecessary. This fact is the inspiration of the newly proposed method based on the compactly supported pdfs.

\subsection{Approximation of the solution of the FPK equation using compactly supported polynomials}

A function has compact support if it is zero outside of a compact set. Let $\Omega_{i}, i=1, \ldots, K$ be the supports of such base functions $\varphi_{\vartheta_{i}}(\cdot)$ parameterized with vectors $\vartheta_{i}$, i.e.

$$
\begin{array}{ll}
\varphi_{\vartheta_{i}}(\mathbf{x}) \neq 0 & \forall \mathbf{x} \in \Omega_{i}, \\
\varphi_{\vartheta_{i}}(\mathbf{x})=0 & \forall \mathbf{x} \notin \Omega_{i},
\end{array}
$$

where $\partial \Omega_{i}$ denotes the boundary of $\Omega_{i}$. The solution of the reduced FPK (10) can be approximately constructed using such compactly supported functions as given in the sequel.

Assume that the pdf which approximate the solution of the reduced FPK (10) is a linear combination of compactly supported functions

$$
p_{\vartheta}(\mathbf{x})=\sum_{i=1}^{K} \kappa_{i} \varphi_{\vartheta_{i}}(\mathbf{x})
$$


where $\kappa_{i} \geqslant 0 \forall i, \sum_{i} \kappa_{i}=1$ and the set of parameters $\vartheta \triangleq\left\{\vartheta_{i}, i=1, \ldots, K\right\}$. In order to fulfill the prerequisites of FPK theorem, it is sufficient that $\varphi_{\vartheta_{i}}(\cdot)$ s satisfy the following conditions for all $i$ :

$$
\begin{aligned}
& \left.\frac{\mathrm{d}^{n} \varphi_{\vartheta_{i}}(\mathbf{x})}{\mathrm{d} \mathbf{x}^{n}}\right|_{\partial \Omega_{i}}=0 \quad \forall n \geqslant 1, \\
& \left.! \exists \mathbf{x}^{*} \in \Omega_{i} \ni \frac{\mathrm{d} \varphi_{\vartheta_{i}}(\mathbf{x})}{\mathrm{d} \mathbf{x}}\right|_{\mathbf{x}=\mathbf{x}^{*}}=0,\left.\quad \frac{\mathrm{d}^{2} \varphi_{\vartheta_{i}}(\mathbf{x})}{\mathrm{d} \mathbf{x}^{2}}\right|_{\mathbf{x}=\mathbf{x}^{*}}<0, \text { and } \\
& \frac{\mathrm{d} \varphi_{\vartheta_{i}}(\mathbf{x})}{\mathrm{d} \mathbf{x}} \neq 0 \quad \forall \mathbf{x} \neq \mathbf{x}^{*}, \\
& \int_{\mathbb{R}^{n}} \varphi_{\vartheta_{i}}(\mathbf{x}) \mathrm{d} \mathbf{x}=\int_{\Omega_{i}} \varphi_{\vartheta_{i}}(\mathbf{x}) \mathrm{d} \mathbf{x}=1 .
\end{aligned}
$$

In other words, $\varphi_{\vartheta_{i}}(\cdot) \mathrm{s}$ are the single bump densities supported in the domain $\Omega_{i}$.

Applying the linear FPK operator to the pdf defined in (17) yields

$$
\mathscr{L} p_{\vartheta}(x)=\mathscr{L} \sum_{i=1}^{K} \kappa_{i} \varphi_{\vartheta_{i}}(x)=\sum_{i=1}^{K} \kappa_{i} \mathscr{L} \varphi_{\vartheta_{i}}(\mathbf{x}) .
$$

Multiplying with the test functions and integrating yields

$$
\int_{\mathbb{R}^{n}} \mathscr{L} p_{\vartheta}(\mathbf{x}) h_{k}(\mathbf{x}) \mathrm{d} \mathbf{x}=\sum_{i=1}^{K} \kappa_{i} \int_{\mathbb{R}^{n}} \mathscr{L} \varphi_{\vartheta_{i}}(\mathbf{x}) h_{k}(\mathbf{x}) \mathrm{d} \mathbf{x}=\sum_{i=1}^{K} \kappa_{i} \int_{\Omega_{i}} \mathscr{L} \varphi_{\vartheta_{i}}(\mathbf{x}) h_{k}(\mathbf{x}) \mathrm{d} \mathbf{x} .
$$

Defining

$$
\Upsilon_{k, i} \triangleq \int_{\Omega_{i}} \mathscr{L} \varphi_{\vartheta_{i}}(\mathbf{x}) h_{k}(\mathbf{x}) \mathrm{d} \mathbf{x}, \quad i=1,2, \ldots K
$$

and using (13), a set of equations in the form,

$$
\begin{aligned}
& \Upsilon_{0} \equiv \sum_{i=1}^{K} \kappa_{i}=1 \\
& \Upsilon_{k} \equiv \sum_{i=1}^{K} \kappa_{i} \Upsilon_{k, i}=0, \quad k=1,2, \ldots,(N-1)
\end{aligned}
$$

is obtained. Solving the system of equations (22) yields the best approximation of the solution where the error between the exact pdf and the approximated pdf vanishes on the space spanned by the test functions $\left\{h_{k}\right\}_{k=1}^{N-1}$.

The essence of the proposed method is to use the compactly supported functions. A family of such functions in the form of compactly supported multivariable polynomials (CSP) which satisfy (18) is given by

$$
\varphi_{\left\{\mu_{i}, \sigma_{i}\right\}_{i=1}^{n}}(\mathbf{x})=\left\{\begin{array}{l}
c \cdot\left(-1+\sum_{i=1}^{n} \frac{\left(x_{i}-\mu_{i}\right)^{2}}{\sigma_{i}^{2}}\right)^{2}, \quad \mathbf{x} \in \Omega_{i}, \\
0, \quad \text { otherwise }
\end{array}\right.
$$

which are supported over the ellipsoidal regions $\Omega_{i}$ defined by

$$
\Omega_{i} \triangleq\left\{\mathbf{x}=\left(x_{1}, \ldots, x_{n}\right)^{\mathrm{T}} \mid \sum_{i=1}^{n} \frac{\left(x_{i}-\mu_{i}\right)^{2}}{\sigma_{i}^{2}} \leqslant 1\right\}
$$

where $\left\{\mu_{i}\right\}_{i=1}^{n}$ and $\left\{\sigma_{i}\right\}_{i=1}^{n}$ are the center and axes lengths of the $n$-dimensional ellipsoids respectively, and the $c$ is the normalization constant chosen according to (18c). With additional $n-1$ parameters, ellipsoidal regions 
can be rotated around the ellipsoid center which results in extra degrees of freedom. The advantage of using such a simple polynomial form of (23) has been demonstrated in Section 3.

\section{Examples}

Following examples chosen from [21] to demonstrate the application and approximation ability of the proposed method.

\subsection{1-D example: bistable system}

Let the system be

$$
\dot{x}=f(x)+\sqrt{\alpha} \eta(t)=\frac{1}{2}\left(x-x^{3}\right)+\sqrt{\alpha} \eta(t)
$$

then the corresponding FPK operator is given by

$$
\mathscr{L} p=\frac{\alpha}{2} \frac{\partial^{2} p}{\partial x^{2}}-\frac{\partial}{\partial x}(f p)=-\frac{1}{2}\left(1-3 x^{2}\right) p(x)-\frac{1}{2}\left(x-x^{3}\right) \frac{\mathrm{d} p(x)}{\mathrm{d} x}+\frac{\alpha}{2} \frac{\mathrm{d}^{2} p(x)}{\mathrm{d} x^{2}} .
$$

The exact solution is known to be [8]

$$
p_{\mathrm{ex}}(x)=\frac{2}{\pi\left(I_{-\frac{1}{4}}\left(\frac{1}{8 \alpha}\right)+I_{\left.\frac{1}{4}\left(\frac{1}{8 \alpha}\right)\right)}\right.} \mathrm{e}^{-\frac{1}{2 \alpha}\left(\frac{1}{4}-x^{2}+\frac{x^{4}}{2}\right)},
$$

where $I_{n}(x)$ is the modified Bessel function of the first kind.

According to the method proposed in Section 2.3, the solution in form of CSP for the stationary density has been assumed in the form of

$$
p_{\mathrm{CSP}}(x)=\sum_{i=1}^{K} \kappa_{i} \varphi_{\mu_{i}, \sigma_{i}}(x)
$$

where the simplest choice for $\varphi_{\vartheta_{i}}$ which satisfies conditions (18) is

$$
\varphi_{\mu_{i}, \sigma_{i}}(x)=\left\{\begin{array}{l}
\frac{15}{16 \sigma_{i}}\left(\left(\frac{x-\mu_{i}}{\sigma_{i}}\right)^{2}-1\right)^{2}, \quad \mu_{i}-\sigma_{i} \leqslant x \leqslant \mu_{i}+\sigma_{i}, \\
0, \quad \text { otherwise }
\end{array}\right.
$$

and it is shown for $\mu_{i}=0, \sigma_{i}=1$ in Fig. 1 .

The total $3 K$ parameters consist of $\vartheta=\left\{\mu_{i}, \sigma_{i}\right\}_{i=1}^{K}$ and $\kappa_{i},(i=1, \ldots, K)$ must be determined from the nonlinear algebraic equations obtained using the described procedure in Section 2.3.

Eq. (21) takes the form

$$
\Upsilon_{k, i}(x)=\int_{\mu_{i}-\sigma_{i}}^{\mu_{i}+\sigma_{i}} \mathscr{L} \varphi_{\mu_{i}, \sigma_{i}}(x) h_{k}(x) \mathrm{d} x
$$

and choosing $h_{k} \triangleq x^{k} k=1, \ldots, 6$, then evaluating (29) yields 


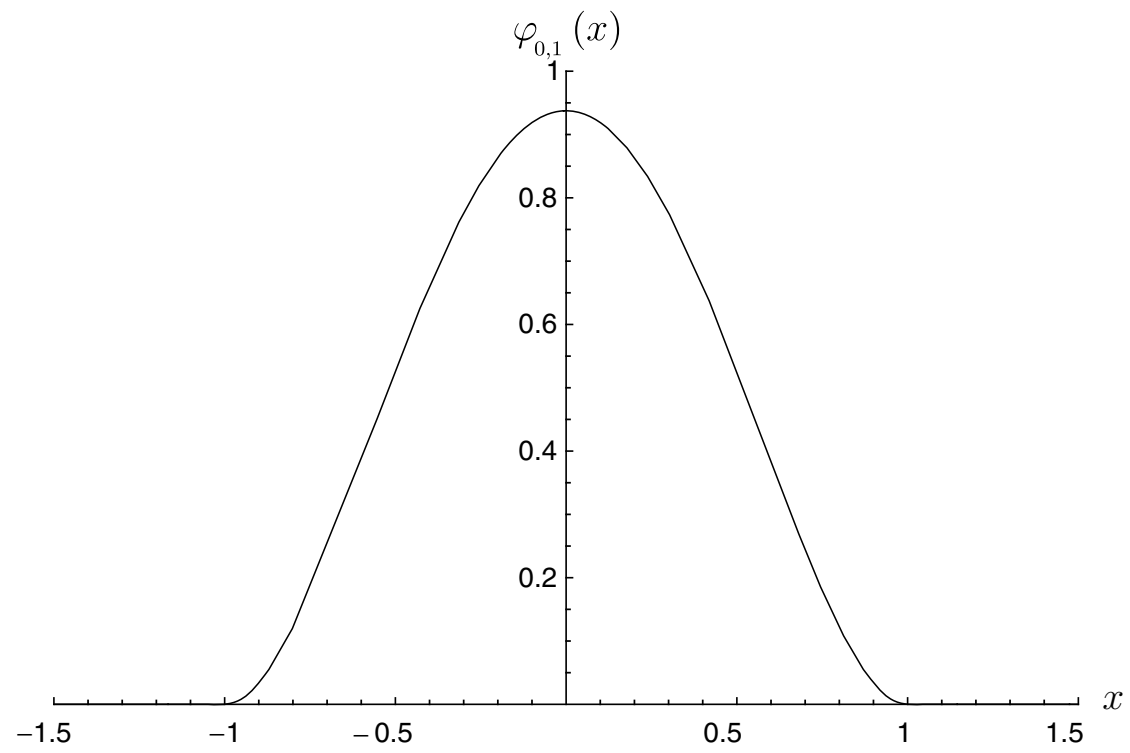

Fig. 1. $\varphi_{0,1}(x)$, the chosen base function for bistable system.

$$
\begin{aligned}
& \Upsilon_{1, i}=\frac{\mu_{i}}{2}-\frac{\mu_{i}^{3}}{2}-\frac{3 \mu_{i} \sigma_{i}^{2}}{14}, \\
& \Upsilon_{2, i}=\alpha+\mu_{i}^{2}-\mu_{i}^{4}+\frac{\sigma_{i}^{2}}{7}-\frac{6 \mu_{i}^{2} \sigma_{i}^{2}}{7}-\frac{\sigma_{i}^{4}}{21}, \\
& \Upsilon_{3, i}=\frac{1}{14} \mu_{i}\left(-21 \mu_{i}^{4}+\left(21-30 \sigma_{i}^{2}\right) \mu_{i}^{2}-5 \sigma_{i}^{4}+9 \sigma_{i}^{2}+42 \alpha\right), \\
& \Upsilon_{4, i}=-2 \mu_{i}^{6}+\left(2-\frac{30 \sigma_{i}^{2}}{7}\right) \mu_{i}^{4}+\frac{2}{7}\left(-5 \sigma_{i}^{4}+6 \sigma_{i}^{2}+21 \alpha\right) \mu_{i}^{2} \cdots+\frac{2}{231}\left(-5 \sigma_{i}^{6}+11 \sigma_{i}^{4}+99 \alpha \sigma_{i}^{2}\right), \\
& \Upsilon_{5, i}=\frac{5}{462} \mu_{i}\left(-231 \mu_{i}^{6}-231\left(3 \sigma_{i}^{2}-1\right) \mu_{i}^{4} \cdots+11\left(-35 \sigma_{i}^{4}+30 \sigma_{i}^{2}+84 \alpha\right) \mu_{i}^{2}-35 \sigma_{i}^{6}+55 \sigma_{i}^{4}+396 \alpha \sigma_{i}^{2}\right), \\
& \Upsilon_{6, i}=-3 \mu_{i}^{8}+\left(3-12 \sigma_{i}^{2}\right) \mu_{i}^{6}+\frac{5}{7}\left(-14 \sigma_{i}^{4}+9 \sigma_{i}^{2}+21 \alpha\right) \mu_{i}^{4} \cdots+\frac{5}{77}\left(-28 \sigma_{i}^{6}+33 \sigma_{i}^{4}+198 \alpha \sigma_{i}^{2}\right) \mu_{i}^{2}-\frac{5 \sigma_{i}^{8}}{143}+\frac{5 \sigma_{i}^{6}}{77}+\frac{5 \alpha \sigma_{i}^{4}}{7} .
\end{aligned}
$$

For the case $K=1,2,3, \alpha=1,2$, and $\kappa_{1}=\kappa_{2}=\kappa_{3}=\frac{1}{3}$ the numerical solutions of (22) and MGC schemes have been enlisted in the Tables 1 and 2, respectively. The comparison of the CSP, MGC schemes for $K=3$ and

\begin{tabular}{|c|c|c|c|c|c|c|}
\hline \multirow[t]{3}{*}{ Parameter } & \multicolumn{6}{|l|}{$K$} \\
\hline & \multicolumn{3}{|l|}{$\alpha=1$} & \multicolumn{3}{|l|}{$\alpha=2$} \\
\hline & 1 & 2 & 3 & 1 & 2 & 3 \\
\hline$\mu_{1}$ & 0.0000 & 0.8645 & 0.0000 & 0.0000 & 0.9261 & 0.0000 \\
\hline$\sigma_{1}$ & 2.5143 & 1.4157 & 2.5207 & 2.8552 & 1.7043 & 2.9343 \\
\hline$\mu_{2}$ & & -0.8645 & 0.9475 & & -0.9261 & 1.0041 \\
\hline$\sigma_{2}$ & & 1.4157 & 1.2442 & & 1.7043 & 1.4919 \\
\hline$\mu_{3}$ & & & -0.9475 & & & -1.0041 \\
\hline$\sigma_{3}$ & & & 1.2442 & & & 1.4919 \\
\hline
\end{tabular}
simulation data has been shown in Fig. 2. The simulation has been done using Euler-Maruyama solver [25] with fixed step size of time increments, $\Delta t=1 \times 10^{-1} \mathrm{~s}$, and $t_{\max }=5020 \mathrm{~s}$, and first $20 \mathrm{~s}$ of the simulation data have been ignored to allow the transients to die out.

Table 1

Numerical solution of nonlinear algebraic equations for the CSP Scheme 
Table 2

Numerical solution of nonlinear algebraic equations for the MGC scheme

\begin{tabular}{|c|c|c|c|c|c|c|}
\hline \multirow[t]{3}{*}{ Parameter } & \multicolumn{6}{|l|}{$K$} \\
\hline & \multicolumn{3}{|l|}{$\alpha=1$} & \multicolumn{3}{|l|}{$\alpha=2$} \\
\hline & 1 & 2 & 3 & 1 & 2 & 3 \\
\hline$\mu_{1}$ & 0.0000 & 0.9388 & 0.0000 & 0.0000 & 0.9261 & 0.0000 \\
\hline$\sigma_{1}$ & 0.8761 & 0.4721 & 0.3346 & 1.0000 & 1.7043 & 0.3675 \\
\hline$\mu_{2}$ & & -0.9388 & 1.1542 & & -0.9261 & 1.2761 \\
\hline$\sigma_{2}$ & & 0.4721 & 0.3837 & & 1.7043 & 0.4629 \\
\hline$\mu_{3}$ & & & -1.1542 & & & -1.2761 \\
\hline$\sigma_{3}$ & & & 0.3837 & & & 0.4629 \\
\hline
\end{tabular}

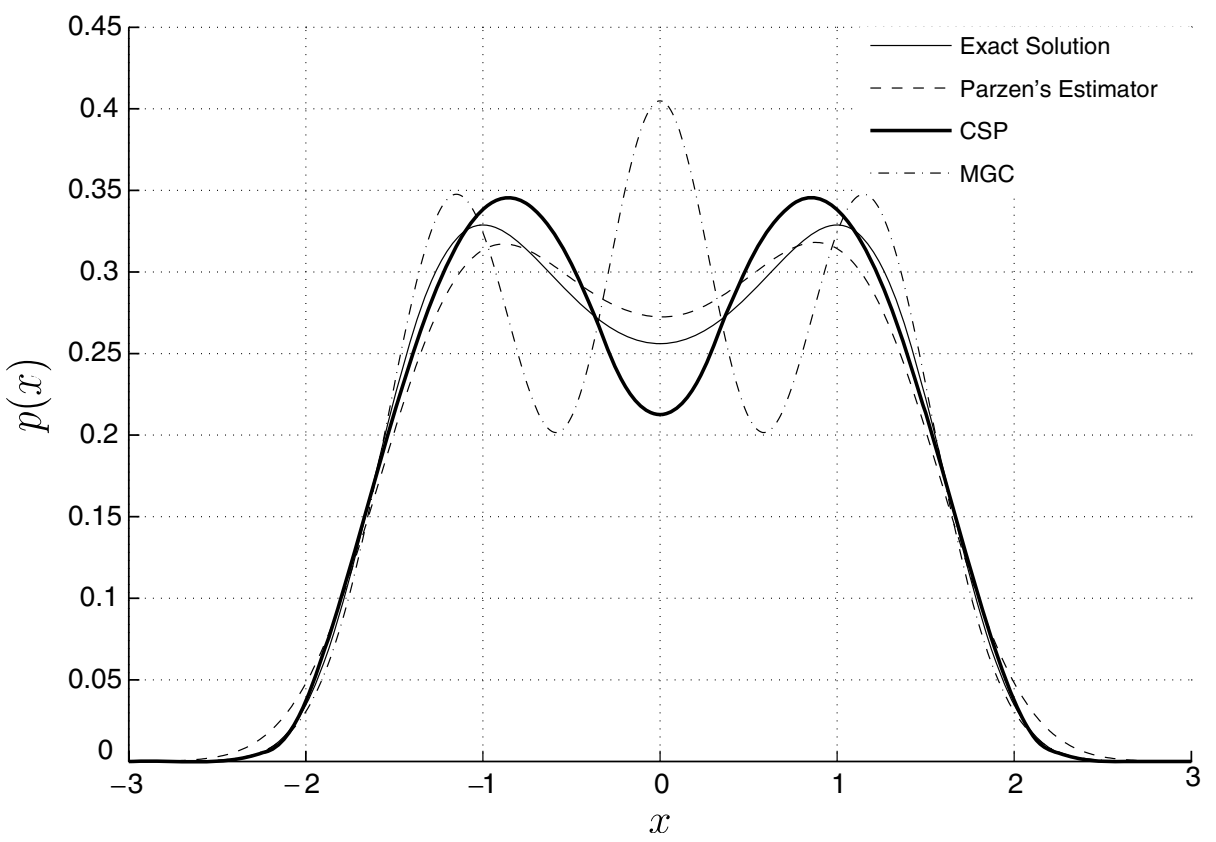

Fig. 2. The pdfs of the state $x$ for 1-D example.

The pdf has been also estimated using the Parzen's estimator [22], i.e.

$$
\hat{p}(x) \triangleq \frac{1}{N h(N)} \sum_{i=1}^{N} g\left(\frac{x-x\left(t_{i}\right)}{h(N)}\right)
$$

with

$$
g(x)=\frac{1}{\sqrt{2 \pi}} \mathrm{e}^{-\frac{x^{2}}{2}}, \quad h(n)=\frac{1}{\sqrt{n}}
$$

and $x\left(t_{i}\right) \triangleq x\left(t_{0}+i \Delta t\right), i=1, \ldots, N$ are the ensemble points obtained from the simulation. The resulting pdfs from the simulations for 100 different realizations of Brownian paths have been averaged to smooth the estimated pdf.

The pdf obtained by the proposed method is very close to the exact solution, and is in agreement with the estimated pdf using the Parzen's estimator from the simulation data.

The error between the approximate and the exact solutions can be determined using the $L_{2}$-norm, i.e. $\left(\|\cdot\|_{2} \triangleq \int(\cdot)^{2} \mathrm{~d} x\right)^{1 / 2}$. The errors between the exact and the approximate solutions have been found by evaluation of $\left\|p_{\mathrm{ex}}(x)-p_{\mathrm{CSP}}(x)\right\|_{2}$ and $\left\|p_{\mathrm{ex}}(x)-p_{\mathrm{MGC}}(x)\right\|_{2}$. They have been shown in Fig. 3 for the case $K=3$. The 
resulting errors for different values of noise amplitudes are smaller for the CSP scheme as compared to MGC scheme.

The moments $E\left(x^{k}\right) \triangleq \int_{\mathbb{R}} x^{k} p(x) \mathrm{d} x, k=1,2, \ldots$, have been evaluated for the exact pdf and the approximate pdfs obtained by CSP and MGC schemes (Table 3), and the relative errors defined as $\left|\frac{E\left(x^{k}\right)-E_{\operatorname{CSP}, M G C}\left(x^{k}\right)}{E\left(x^{k}\right)}\right| \times 100$ have been calculated for $\alpha=1,2$ and $K=3$. The relative errors for $\alpha=1$ are then compared. Clearly, the resulting error profile is better for the CSP scheme as can be seen in Fig. 4.

The resulting approximate pdfs of CSP scheme, as depicted in Fig. 5, are in agreement with the expected physical situation as the noise power $\alpha$ is changed. When the noise power is small, the probability of finding the state near the stable equilibria of the system with $\alpha=0$ will be higher. As the noise power increases, the

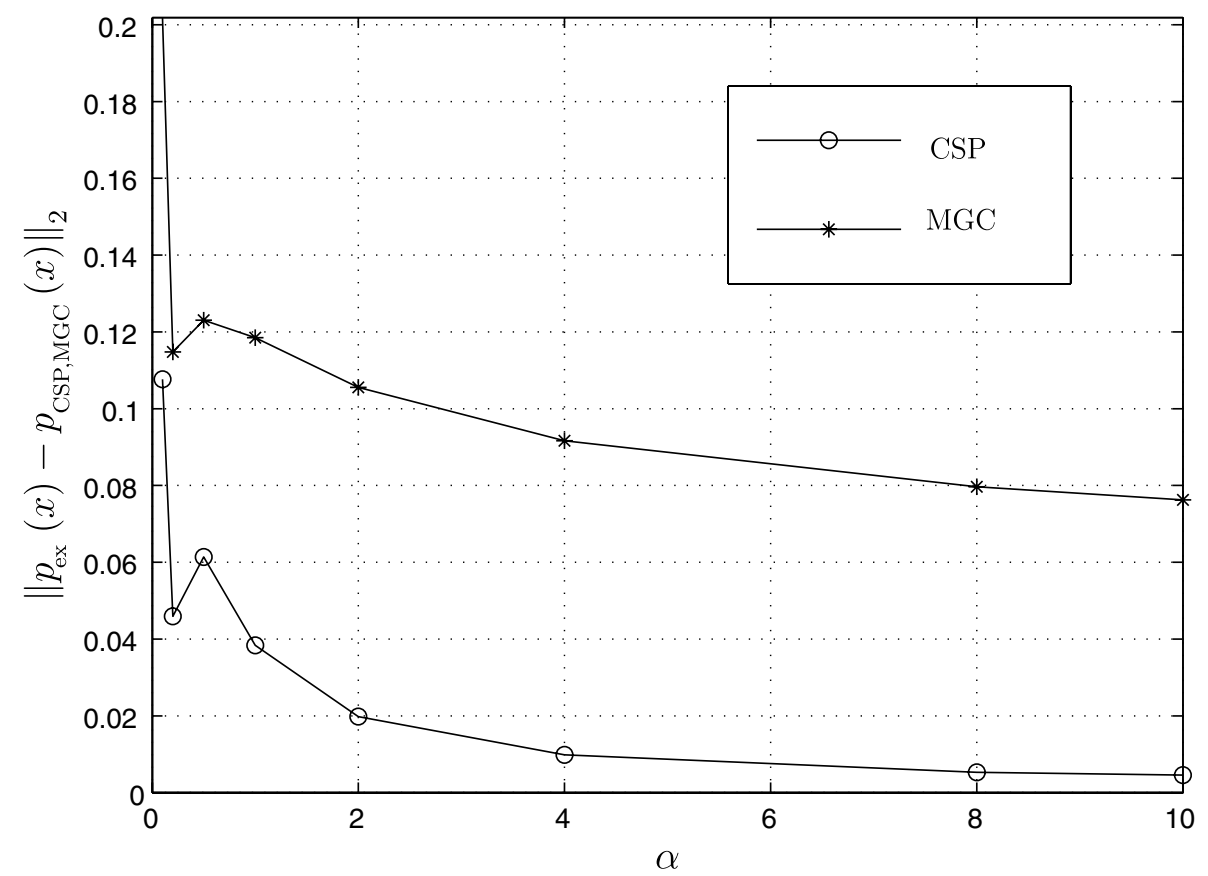

Fig. 3. Comparison of $\left\|p_{\mathrm{ex}}(x)-p_{\mathrm{CSP}}(x)\right\|_{2}$ and $\left\|p_{\mathrm{ex}}(x)-p_{\mathrm{MGC}}(x)\right\|_{2}$ for various values of $\alpha$ and $K=3$.

Table 3

Comparison of the moments for the exact and the approximate solutions

\begin{tabular}{|c|c|c|c|c|c|c|}
\hline \multirow[t]{2}{*}{$\alpha$} & \multirow[t]{2}{*}{$k$} & \multicolumn{3}{|l|}{ Moments } & \multicolumn{2}{|c|}{ Relative error } \\
\hline & & $\overline{E_{\mathrm{ex}}\left(x^{k}\right)}$ & $E_{\mathrm{MGC}}\left(x^{k}\right)$ & $\overline{E_{\mathrm{CSP}}\left(x^{k}\right)}$ & $\overline{\mathrm{MGC}}$ & CSP \\
\hline \multirow[t]{6}{*}{1} & odd & 0 & 0.0000 & 0.0000 & - & - \\
\hline & 2 & 1.0418 & 1.0236 & 1.0486 & 1.7460 & 0.6494 \\
\hline & 4 & 2.0418 & 2.0236 & 2.0486 & 0.8908 & 0.3313 \\
\hline & 6 & 5.1672 & 5.0944 & 5.1942 & 1.4081 & 0.5237 \\
\hline & 8 & 15.3762 & 15.2125 & 15.4371 & 1.0647 & 0.3960 \\
\hline & 10 & 51.5465 & 52.1506 & 51.3131 & 1.1720 & 0.4528 \\
\hline \multirow[t]{6}{*}{2} & odd & 0 & 0.0000 & 0.0000 & - & - \\
\hline & 2 & 1.2905 & 1.2734 & 1.2942 & 1.3214 & 0.2868 \\
\hline & 4 & 3.2905 & 3.2734 & 3.2942 & 0.5182 & 0.1125 \\
\hline & 6 & 11.0333 & 10.9139 & 11.0592 & 1.0819 & 0.2348 \\
\hline & 8 & 43.9379 & 43.6480 & 44.0008 & 0.6598 & 0.1432 \\
\hline & 10 & 198.4034 & 201.9996 & 197.5986 & 1.8126 & 0.4057 \\
\hline
\end{tabular}




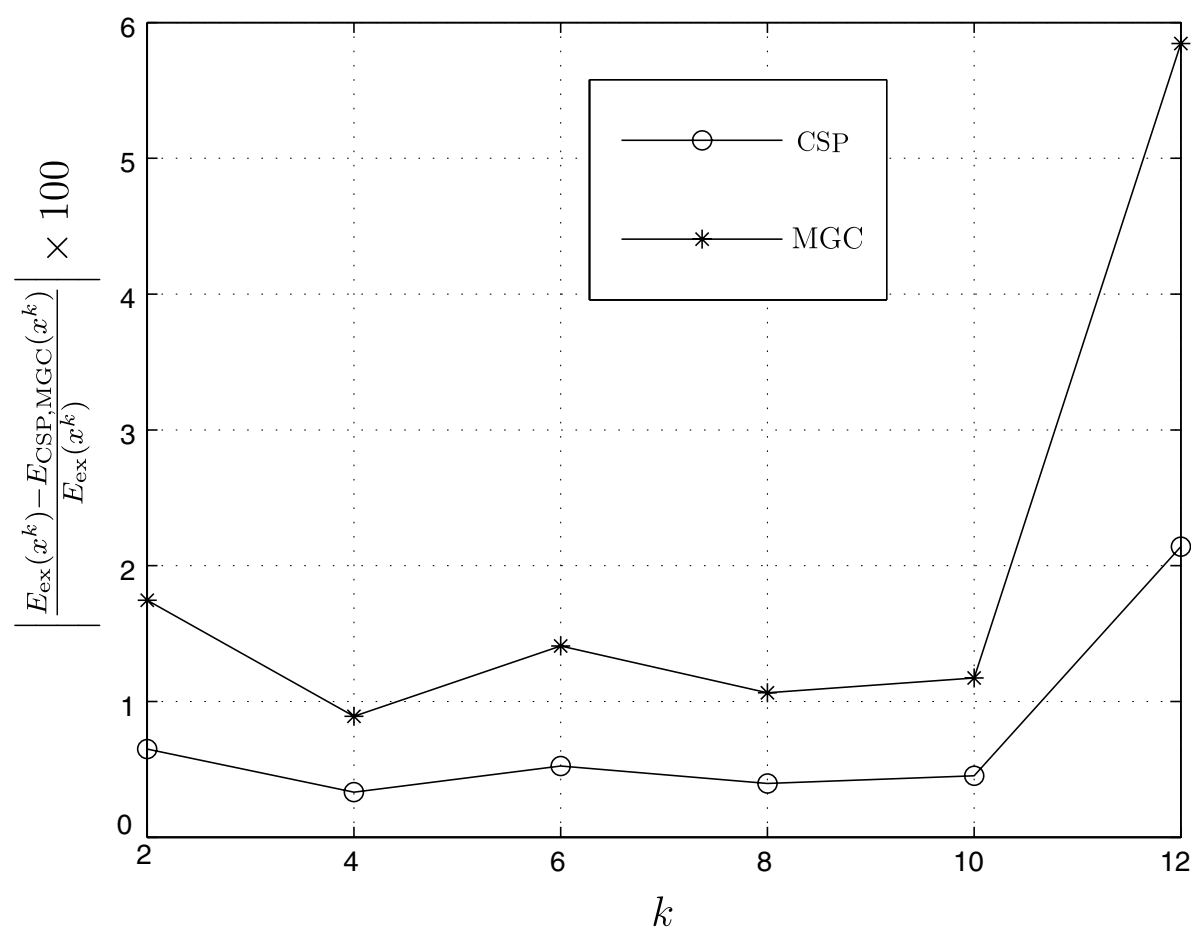

Fig. 4. Comparison of the relative errors with respect to the exact moments for MGC and CSP schemes $(\alpha=1)$.

peaks centered about the equilibria broadens, which implies that when averaged, the trajectory switches more frequently between the stable equilibria.

\subsection{2-D example: duffing system under the Gaussian white noise excitation}

Let the system (1) be as

$$
\left(\begin{array}{l}
\dot{x} \\
\dot{y}
\end{array}\right)=\left(\begin{array}{c}
y \\
-x-\epsilon x^{3}-\alpha y
\end{array}\right)+\left(\begin{array}{cc}
0 & 0 \\
0 & \sqrt{2 \alpha}
\end{array}\right)\left(\begin{array}{l}
\eta_{0} \\
\eta_{1}
\end{array}\right),
$$

where $\eta_{0}(t), \eta_{1}(t)$ are one-dimensional independent Gaussian white noises. Corresponding FPK operator is given by

$$
\mathscr{L}_{\epsilon, \alpha} p=\alpha p+\left(x+\epsilon x^{3}+\alpha y\right) \frac{\partial p}{\partial y}-y \frac{\partial p}{\partial x}+\alpha \frac{\partial^{2} p}{\partial y^{2}} .
$$

The exact solution of the (32) is known to be [8]

$$
p_{\text {ex }}(x, y)=\frac{\sqrt{\epsilon}}{\sqrt{\pi} K_{\frac{1}{4}}\left(\frac{1}{8 \epsilon}\right)} \mathrm{e}^{\frac{-1}{8 \epsilon}-\frac{x^{2}}{2}-\frac{y^{2}}{2}-\frac{e^{4}}{4}}
$$

where $K_{n}(x)$ denotes the modified Bessel functions of the second kind. Note that the states are scaled so that the exact pdf does not depend on $\alpha$.

The base function $\varphi_{\vartheta_{i}}$ has been chosen as

$$
\varphi_{a_{i}, b_{i}, \mu_{1 i}, \mu_{2 i}}(x, y)=\left\{\begin{array}{l}
\frac{3}{\pi a_{i} b_{i}}\left(\left(\frac{x-\mu_{1 i}}{a_{i}}\right)^{2}+\left(\frac{y-\mu_{2 i}}{b_{i}}\right)^{2}-1\right)^{2}, \quad\left(\frac{x-\mu_{i 1}}{a_{i}}\right)^{2}+\left(\frac{y-\mu_{i 2}}{b_{i}}\right)^{2} \leqslant 1, \\
0, \quad \text { otherwise. }
\end{array}\right.
$$



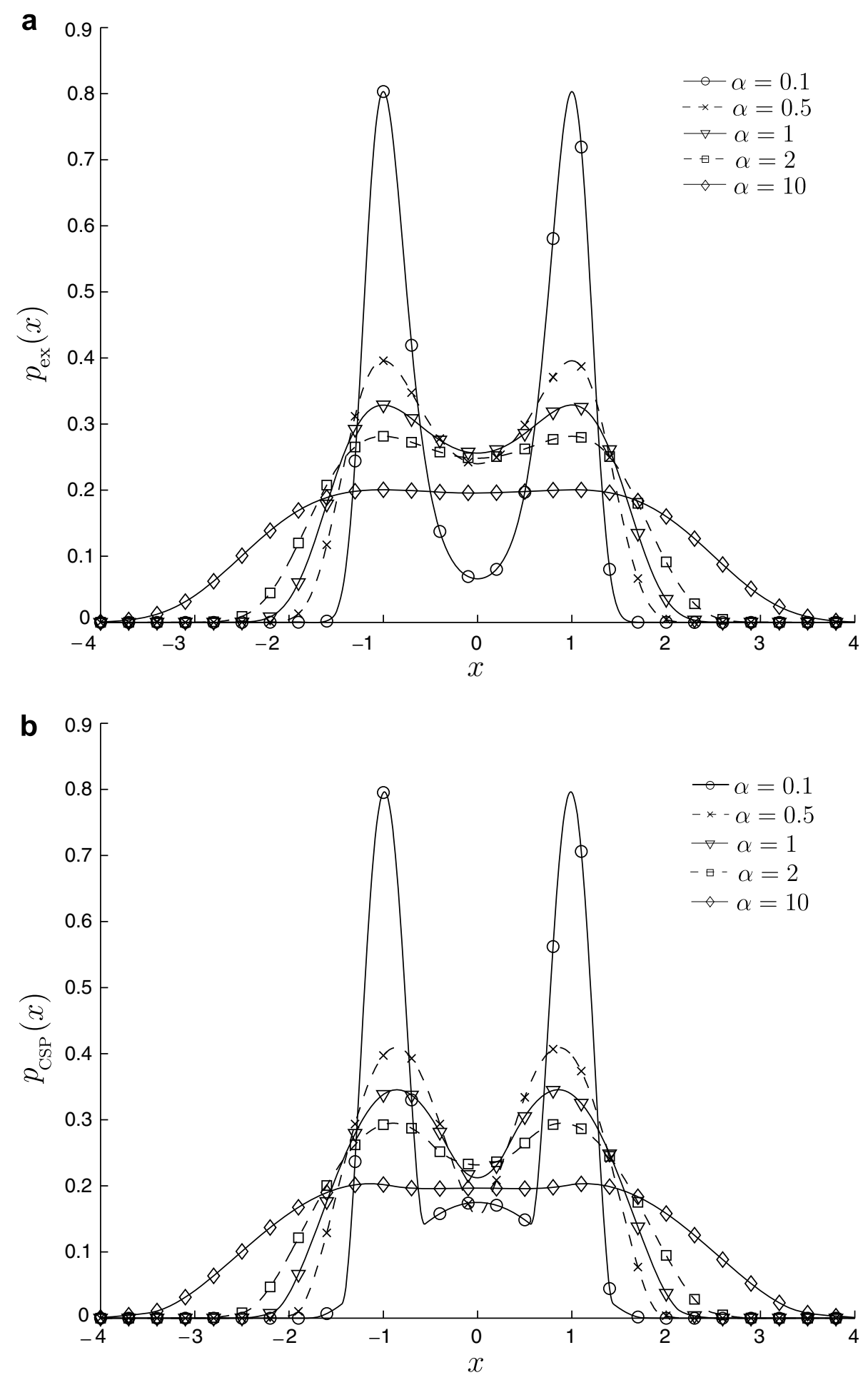

Fig. 5. The effect of increasing noise power on the approximate pdf for $K=3$ for the bistable 1-D system compared to exact solution: (a) exact pdf; (b) CSP pdf.

The function $\varphi_{a_{i}, b_{i}, \mu_{1 i}, \mu_{2 i}}$ is compactly supported inside elliptic region defined by $\Omega_{i}=\left\{x, y \in \mathbb{R} \mid\left(\frac{x-\mu_{i 1}}{a_{i}}\right)^{2}+\right.$ $\left.\left(\frac{y-\mu_{i 2}}{b_{i}}\right)^{2} \leqslant 1\right\}$. 
Assuming that the solution is in the form

$$
p_{\mathrm{CSP}}(x, y)=\sum_{i=1}^{K} \kappa_{i} \varphi_{a_{i}, b_{i}, \mu_{i}, \mu_{2 i}}(x, y),
$$

$5 K$ parameters (i.e., $\vartheta=\left\{a_{i}, b_{i}, \mu_{1 i}, \mu_{2 i}\right\}_{i=1}^{K}$ and $\left\{\kappa_{i}\right\}_{i=1}^{K}$ ) should be determined. The test functions have been chosen as $h_{1}(x, y)=x, h_{2}(x, y)=y, h_{3}(x, y)=x y, h_{4}(x, y)=x^{2}$ and $h_{5}(x, y)=y^{2}$. In order to simplify the integrals in (21) the following transformation

$$
\begin{aligned}
& x \rightarrow \mu_{1 i}+a_{i} r \cos (\phi), \\
& y \rightarrow \mu_{i 2}+b_{i} r \sin (\phi),
\end{aligned}
$$

which transformed the elliptic regions to the region inside the unit circle have been specifically chosen. Evaluating,

$$
\Upsilon_{k, i}=a_{i} b_{i} \int_{-\pi}^{\pi} \int_{0}^{1} \mathscr{L}_{\epsilon, \alpha} \varphi_{a_{i}, b_{i}, \mu_{1 i}, \mu_{2 i}}(r, \phi) h_{k}(r, \phi) r \mathrm{~d} r \mathrm{~d} \phi
$$

for $k=1, \ldots, 5$ yields

$$
\begin{aligned}
& \Upsilon_{1, i}=\mu_{2 i} \\
& \Upsilon_{2, i}=-\frac{1}{8} \mu_{1 i}\left(3 \epsilon a_{i}^{2}+8 \epsilon \mu_{1 i}^{2}+8\right)-\alpha \mu_{2, i}, \\
& \Upsilon_{3, i}=\frac{1}{80}\left(-3 \epsilon a_{i}^{4}-10\left(6 \epsilon \mu_{1 i}^{2}+1\right) a_{i}^{2}+10\left(b_{i}^{2}-8\left(\epsilon \mu_{1 i}^{4}+\mu_{1 i}^{2}+\alpha \mu_{2 i} \mu_{1 i}-\mu_{2 i}^{2}\right)\right)\right), \\
& \Upsilon_{4, i}=2 \mu_{1 i} \mu_{2 i}, \\
& \Upsilon_{5, i}=\frac{1}{4}\left(-\left(b_{i}^{2}+8 \mu_{2, i}^{2}\right) \alpha+8 \alpha-\mu_{1 i}\left(3 \epsilon a_{i}^{2}+8 \epsilon \mu_{1 i}^{2}+8\right) \mu_{2 i}\right) .
\end{aligned}
$$

For a first approximation with $K=1$, solving (22) gives

$$
\mu_{11}=\mu_{21}=0, \quad a_{1}=\sqrt{\frac{\sqrt{5} \sqrt{48 \epsilon+5}}{3 \epsilon}-\frac{5}{3 \epsilon}}, \quad b_{1}=2 \sqrt{2} .
$$

This solution has been depicted in Fig. 6, for $\epsilon=0.1,1,10$. At the same resolution, MGC scheme with the parameters

$$
\mathbf{m}_{1}=\left(\mu_{11} \mu_{21}\right)^{\mathrm{T}}, \quad \Gamma_{1}=\left(\begin{array}{cc}
\sigma_{11}^{2} & \rho_{11} \sigma_{11} \sigma_{21} \\
\rho_{11} \sigma_{11} \sigma_{21} & \sigma_{21}^{2}
\end{array}\right)^{\mathrm{T}}
$$

in (14) results in

$$
\mu_{11}=\mu_{21}=\rho_{11}=0, \quad \sigma_{11}=\sqrt{\frac{\sqrt{12 \epsilon+1}}{6 \epsilon}-\frac{1}{6 \epsilon}}, \quad \sigma_{21}=1 .
$$

A comparison of exact solution, the solution for the MGC, and proposed schemes for the marginal density $p(x)$ are shown in Fig. 7. To investigate the effect of increasing nonlinearity on the approximation error, $\left\|p_{\mathrm{ex}}(x, y)-p_{\mathrm{CSP}}(x, y)\right\|_{2}$ and $\left\|p_{\mathrm{ex}}(x, y)-p_{\mathrm{MGC}}(x, y)\right\|_{2}$ have been compared in Fig. 8.

$\epsilon=0$ corresponds to a linear systems for which the state pdf is purely Gaussian, hence the pdf calculated using the MGC scheme is exact. However, due to the filtering introduced by the compactly supported polynomials by truncating the pdf at the tails, the error is high for the CSP scheme. As the value of $\epsilon$ increases, the approximation error introduced by the MGC scheme increases fast, where as the approximation error of CSP scheme scales almost linearly. Hence, when the system is highly nonlinear, the CSP scheme results in better approximation error profile.

The proposed scheme estimates the pdf of the states in both examples successfully. Using the compactly supported polynomials as in (23) has an advantage that it needs at most $3 K n$ parameters to be determined 

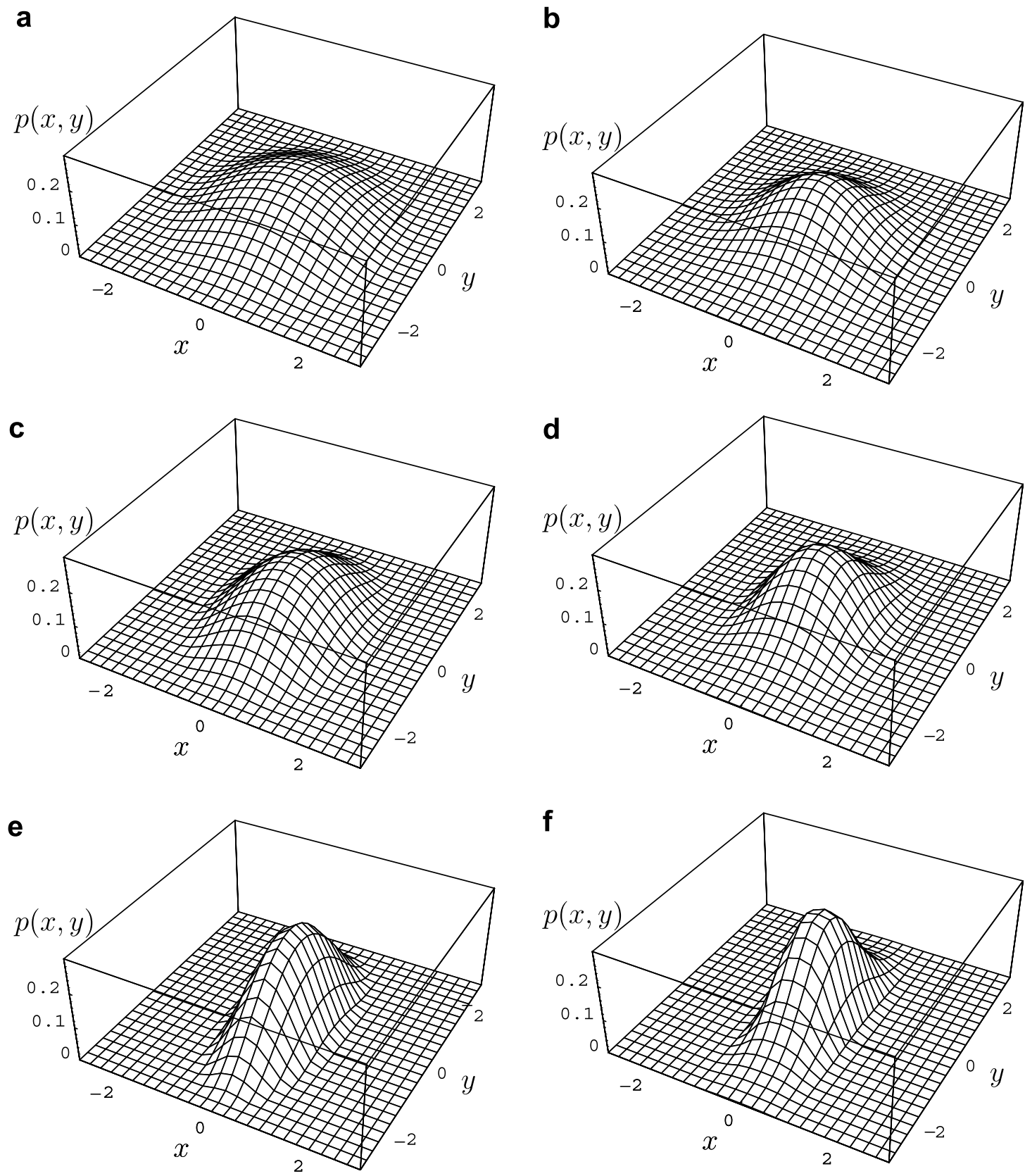

Fig. 6. $p_{a_{1}, b_{1}, \mu_{11}, \mu_{21}}(x, y)$, the CSP solution of duffing system for $K=1$ : (a) CSP, $\epsilon=0.1$; (b) exact, $\epsilon=0.1$; (c) CSP, $\epsilon=1$; (d) exact, $\epsilon=1$; (e) CSP, $\epsilon=10$; (f) exact, $\epsilon=10$.

totally (i.e., $n, n, n-1$ parameters for axis lengths, the center point and the axis rotations of the $n$-dimensional ellipsoids, respectively, together with the weights of the base functions). On the other hand, Multi-Gaussian Closure scheme needs $K \frac{(n+2)(n+1)}{2}$ parameters, in the same resolution. Further reduction in number of parameters to be determined is possible if the ellipsoid axes are chosen to be equal and the rotations are omitted, which may be suitable in some cases as in example 2. Besides, there is an obvious numerical advantage that the integrations over the ellipsoidal regions are quite easier, e.g. to calculate needed the moments.

On the other hand, because of the properties of the base functions, the density obtained by the proposed method can not approximate the tail of the exact pdfs well. Hence, the proposed form of approximate densities 


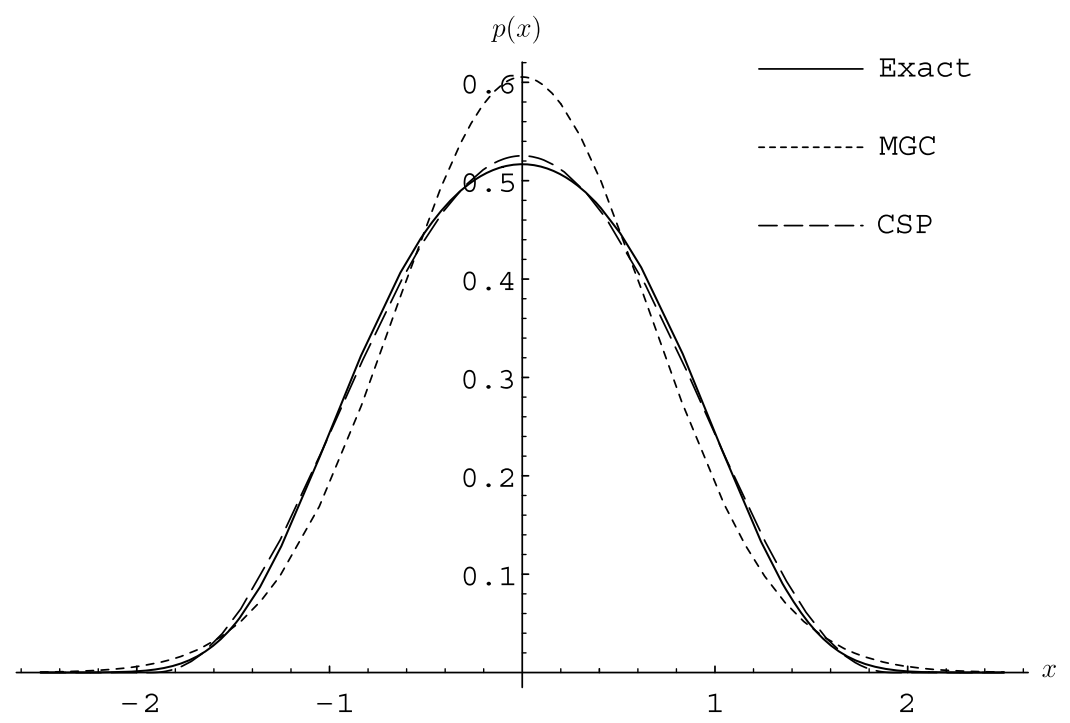

Fig. 7. A comparison of marginal densities obtained by exact, MGC and CSP solutions for the noise driven duffing system. $K=1, \epsilon=1$.

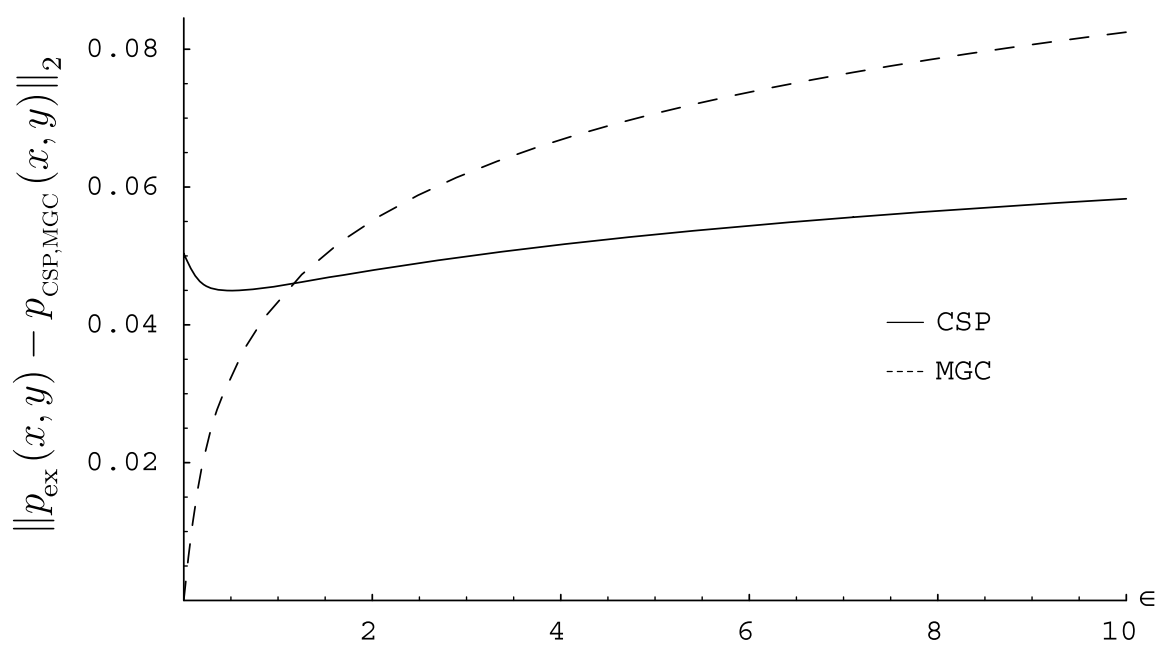

Fig. 8. The effect of increasing nonlinearity on the approximation error.

may not be suitable for some cases such as reliability analysis. The proposed method is more suitable when qualitative behavior of the system under consideration is to be characterized. The approximate solutions obtained can also be used as an initial condition for iterative FPK solution methods as in [11] to improve the accuracy.

\section{Conclusions}

In this paper, the new method of obtaining set of nonlinear algebraic equations whose solutions are the parameters of compactly supported approximate solution of FPK equation in the weak sense for the nonlinear systems driven with Gaussian white noise, has been presented. With the specific choice of compactly supported polynomial functions, it has been possible to choose the number of parameters to be determined considerably smaller than the MGC scheme as the dimensionality of the system increases. The errors between the exact solution and approximate solutions have been examined, and compared for the two examples commonly 
encountered in the literature. The pdfs obtained by the proposed method are very close to the exact solution, and are in agreement with the pdfs estimated by the Parzen's estimator obtained from the simulation data. The approximate pdfs of the CSP method also confirm the expected physical behavior as the noise power is changed. The method can also be used to determine stationary densities arising from the chaotic behavior of the low dimensional deterministic dynamical systems due to the uncertainties in the initial conditions. Since the calculation of the related entropies in terms of the systems parameters are possible with the determination of the densities, the entropy increase in the bifurcation phenomena can also be observed for such systems. Hence, as a future work, the controlling the chaotic dynamics might be achieved by optimization of the entropies calculated via the pdfs using the CSP scheme.

\section{Acknowledgements}

Research is supported by the Research Council KUL: GOA-AMBioRICS, CoE EF/05/006 Optimization in Engineering, Flemish Government: FWO: G.0211.05 (Nonlinear Systems), G.0226.06 (Cooperative Systems) Belgian Federal Science Policy Office IUAP P5/22 ('Dyn. Sys. and Ctrl.: Computation, Identification and Modeling') and Turkish Scientific Research and Development Council (TUBITAK).

\section{References}

[1] H. Risken, The Fokker-Planck Equation: Methods of Solution and Applications, second ed., Springer-Verlag, Berlin, Germany, 1996.

[2] A. Lasota, M.C. Mackey, Chaos, Fractals and Noise: Stochastic Aspects of Dynamics, second ed.Applied Mathematical Sciences, Springer-Verlag, Berlin, Germany, 1994.

[3] R. Wang, K. Yasuda, Z. Zhang, A generalized analysis technique of the stationary FPK equation in nonlinear systems under Gaussian white noise excitations, International Journal of Engineering Science 38 (12) (2000) 1315-1330.

[4] R. Wang, K. Yasuda, Exact stationary probability density for second order nonlinear systems under external white noise excitation, Journal of Sound and Vibration 205 (5) (1997) 647-655.

[5] G.Q. Cai, Y.K. Lin, Exact and approximate solutions for randomly excited MDOF non-linear systems, International Journal of NonLinear Mechanics 31 (5) (1996) 647-655.

[6] T. Caughey, F. Ma, The exact solution of a class of nonlinear stochastic systems, International Journal of Non-Linear Mechanics 17 (1982) 137-142.

[7] M. Dimentberg, An exact solution to a certain nonlinear random vibration problem, International Journal of Non-Linear Mechanics 17 (1982) 231-236.

[8] T.T. Soong, Random Differential Equations in Science and Engineering, Academic Press, New York, 1973.

[9] J. Johnson, R. Scott, Extension of eigenfunction-expansion solution to a Fokker-Planck equation-II. Second order systems, International Journal of Non-Linear Mechanics 15 (1980) 41-56.

[10] J.D. Atkinson, Eigenfunction expansions for randomly excited nonlinear systems, Journal of Sound and Vibrations 30 (1973) $153-$ 172.

[11] W. Mayfield, A sequence solution to the Fokker-Planck equation, IEEE Transactions on Information Theory 19 (2) (1973) $165-176$.

[12] E.T. Jaynes, Information theory and statistical mechanics, Physical Review 106 (4) (1957) 620-630.

[13] S.A. El-Wakil, E.M. Abulwafa, M.A. Abdou, A. Elhanbaly, Maximum-entropy approach with higher moments for solving FokkerPlanck equation, Physica A: Statistical Mechanics and its Applications 315 (3-4) (2002) 480-492.

[14] S.A. El-Wakil, A. Elhanbaly, M.A. Abdou, Solution of Fokker-Planck equation by means of maximum entropy approach, Journal of Quantitative Spectroscopy and Radiative Transfer 69 (1) (2001) 41-48.

[15] U.V. Wagner, W.V. Wedig, On the calculation of stationary solutions of multi-dimensional Fokker-Planck equations by orthogonal functions, Nonlinear Dynamics 21 (3) (2000) 286-306.

[16] G.-K. Er, Exponential closure method for some randomly excited non-Linear systems, International Journal of Nonlinear Mechanics 35 (1) (2000) 69-78.

[17] G.-K. Er, A consistent method for the solution to reduced FPK equation in statistical mechanics, Physica A: Statistical and Theoretical Physics 262 (1-2) (1999) 118-128.

[18] Q. Liu, H.G. Davies, The non-stationary response probability density functions of non-linearly damped oscillators subjected to white noise excitations, Journal of Sound and Vibration 139 (3) (1990) 425-435.

[19] C. Soize, Steady-state solution of Fokker-Planck equation in higher dimension, Probabilistic Engineering Mechanics 3 (4) (1988) 196206.

[20] R.N. Iyengar, P.K. Dash, Study of the random vibration of nonlinear systems by the gaussian closure technique, Journal of Applied Mechanics 45 (1978) 393-399.

[21] G.-K. Er, Multi-Gaussian closure method for randomly excited nonlinear systems, International Journal of Non-Linear Mechanics 33 (2) (1998) 201-204. 
[22] K.S. Shanmugan, A.M. Breipohl, Random Signals: Detection, Estimation and Data Analysis, John Wiley and Sons, New York, USA, 1988.

[23] M.P. Wand, M.C. Jones, Kernel Smoothing, Chapman and Hall, London, 1995.

[24] M.D. Paola, A. Sofi, Approximate solution of the Fokker-Planck-Kolmogorov equation, Probabilistic Engineering Mechanics 17 (4) (2002) 369-384.

[25] P.E. Kloeden, E. Platen, Numerical Solution of Stochastic Differential Equations, Springer Verlag, 2000. 